\title{
Attention/memory complaint is correlated with motor speech disorder in Parkinson's disease
}

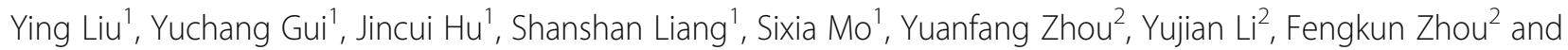
Jianwen $\mathrm{Xu}^{1 *}$

\begin{abstract}
Background: The mechanisms underlying the online modulation of motor speech in Parkinson's disease (PD) have not been determined. Moreover, medical and rehabilitation interventions for PD-associated motor speech disorder (MSD) have a poor long-term prognosis.

Methods: To compare risk factors in PD patients with MSD to those without MSD (non-MSD) and determine predictive independent risk factors correlated with the MSD phenotype, we enrolled 314 PD patients, including 250 with and 64 without MSD. We compared demographic, characteristic data, as well as PD-associated evaluations between the MSD group and non-MSD group.

Results: Univariate analysis showed that demographic characteristics, including occupation, educational level, monthly income and speaking background; clinical characteristics, including lesions in the frontal and temporal lobes, and concurrent dysphagia; and PD-associated evaluations, including the activity of daily living (ADL) score, non-motor symptoms scale (NMSS) domain 4 score (perceptual problem), and NMSS domain 5 score (attention/ memory) were all significantly different between the MSD and non-MSD group (all $P<0.05$ ). Multivariate logistic regression analysis showed that educational level, frontal lesions, and NMSS domain 5 score (attention/memory) were independent risk factors for PD-associated MSD (all $P<0.005$ ).
\end{abstract}

Conclusions: We determined an association between MSD phenotype and cognitive impairment, reflected by lowlevel education and related clinical profiles. Moreover, attention and memory dysfunction may play key roles in the progression of MSD in PD patients. Further studies are required to detail the mechanism underlying abnormal speech motor modulation in PD patients. Early cognitive intervention may enhance rehabilitation management and motor speech function in patients with PD-associated MSD.

Keywords: Motor speech disorder, Parkinson's disease, Cognitive, NMSS

\section{Introduction}

Parkinson's disease (PD) is a common movement disorder that occurs in more than $1 \%$ of the population worldwide, especially in those over the age of 60 years. PD gives rise to many different kinds of abnormal motor manifestations [1]. As a slow progressing neurodegenerative disease that involves apoptosis of non-dopaminergic neurons, PD imposes various levels of non-motor manifestations (NMM)

\footnotetext{
* Correspondence: xujianwen@gxmu.edu.cn

${ }^{1}$ Department of Rehabilitation Medicine, the First Affiliated Hospital of

Guangxi Medical University, Nanning 530021, Guangxi, China

Full list of author information is available at the end of the article
}

burden on both young and old individuals, resulting in difficulty in daily activities and social support [2]. Emerging evidence has found that NMM can occur in early or late stage PD and across all stages of life [2, 3]. Moreover, some NMM, including autonomic symptoms, cognitive impairment, mood, and sensory dysfunction, can influence the progression of motor symptoms $[4,5]$.

Although abnormal motor control of the limbs and trunk is common among PD patients, motor speech disorder (MSD) is also experienced by almost $90 \%$ of the patients [6]. MSD, which typically manifests as reduced vocal frequency and volume, as well as abnormal control

(C) The Author(s). 2019 Open Access This article is distributed under the terms of the Creative Commons Attribution 4.0 International License (http://creativecommons.org/licenses/by/4.0/), which permits unrestricted use, distribution, and 
of the motor aspects of speech, is regarded as a serious impediment to individual psychological health and family care. As a result, MSD is a critical negative determinant of the quality of life of PD patients [7]. Currently, routine speech therapy and medical interventions using anti-PD drugs are the two main therapeutic approaches for PD-associated MSD. In the past, routine speech therapy, including respiratory, voice, and tuning training, as well as rhythmic training, was conventionally carried out for PD-associated MSD. However, the fact that routine speech therapy is mainly aimed at the articulation organs and muscles, rather than the root lesions in the neural substrates responsible for speech motor control, makes it a short-term approach and less effective $[8,9]$. On the other hand, given that lesions responsible for PDassociated MSD may not only be found in the basal ganglia, there is mounting evidence that medical interventions are ineffective and may even worsen MSD in PD patients [10-12]. Therefore, the current interventions for PD-associated MSD are still insufficient. This may be attributed to insufficient information on the primary cause of PD-associated MSD, with the pathophysiological mechanisms underlying abnormal motor speech modulation in PD not yet understood.

Speech motor control is predominantly under the online modulation of auditory feedback, which is encoded mainly by cortical areas, especially the frontal and temporal lobes [13, 14]. The frontal and temporal areas are closely associated with high-level cognitive functions, such as attention and working memory, which play important roles in speech motor control $[15,16]$. Moreover, several studies have reported that cognitive functions could compensate for auditory degeneration resulting from aging and the diseased brain [17]. Therefore, these high-level cognitive functions may be the target for studies on the pathophysiological mechanism underlying abnormal motor speech modulation as well as the development of novel rehabilitation approaches for PD-associated MSD. Nonetheless, there have been no reports on whether the MSD phenotype is associated with high-level cognitive impairment.

Cognitive impairment has recently been shown to be a risk factor for other kinds of speech disorders (SD) [18], therefore, we speculated that patients with $\mathrm{PD}$-associated MSD might experience a greater NMM burden, especially more severe cognitive impairment. However, there is currently limited evidence on whether the MSD group experiences a greater NMM burden than the non-MSD group or not. To improve the current gaps in the clinical data, we assessed probable independent risk factors, including demographic and clinical characteristics and non-motor symptom scale (NMSS) scores. We aimed to improve the literature on PD-associated MSD and assist future studies on its pathophysiological mechanism. Moreover, we sought to provide information that may help in the development of novel rehabilitation protocols and improvement of current ones.

\section{Methods \\ Patients}

We enrolled 314 patients (age range: $42-83$ years) with PD. All of them were hospitalized or accepted outpatient service in department of neurology or rehabilitation medicine, within the First Affiliated Hospital of Guangxi Medical University. Two neurologists independently confirmed the diagnosis of PD based on the United Kingdom PD Brain Bank's definition [19]. The exclusion criteria included: (1) clinically confirmed diagnosis of PD secondary to other diseases; (2) PD concurrent with severe systemic debilitating diseases, such as cardiac, renal, or liver failure; and (3) incomplete case history and neuroimaging data. This study was approved by the Ethics Committee of the First Affiliated Hospital of Guangxi Medical University. We obtained informed consent from each patient.

The clinical diagnosis of MSD was confirmed using scores of both the speech (oral communication) item (item 18) of the Unified Parkinson's Disease Rating Scale (UPDRS) and the Frenchay dysarthria assessment (FDA) by two separate speech therapists. In UPDRS-item 18, score 0 denotes a normal speech motor, Score 1 denotes a mild decline of oral communication and vocal frequency, score 2 denotes to a moderate decline of oral communication and vocal frequency, score 3 denotes to a remarkable decline of oral communication and vocal frequency, and score 4 denotes to a disability of speech motor. Patients with a score 1 or higher were considered as having MSD. In FDA, there are 28 evaluation items. Grade a denotes to a completely normal status. The final evaluated score was the Grade a score and patients with a score of 26a or lower were considered as having MSD. MSD diagnosis was only confirmed when the patient had both an UPDRS-item 18 score of one or higher and an FDA score of 26a or lower. Based on these two scales, we assigned the PD patients to either the MSD or nonMSD group. In this study, there were 250 patients in the MSD group and 64 patients in the non-MSD group. The whole diagnostic procedure of MSD is shown in Fig. 1.

\section{Data collection and scale evaluation}

Demographic data were collected via face-to-face interviews or written questionnaires. The mental labor was mainly characterized by application of intelligence, scientific and cultural knowledge and production skills during their labor. And the manual labor was mainly characterized by application of physical strength, production tool and service during their labor.

Clinical data, including case history and magnetic resonance imaging (MRI) brain scans, were collected from 


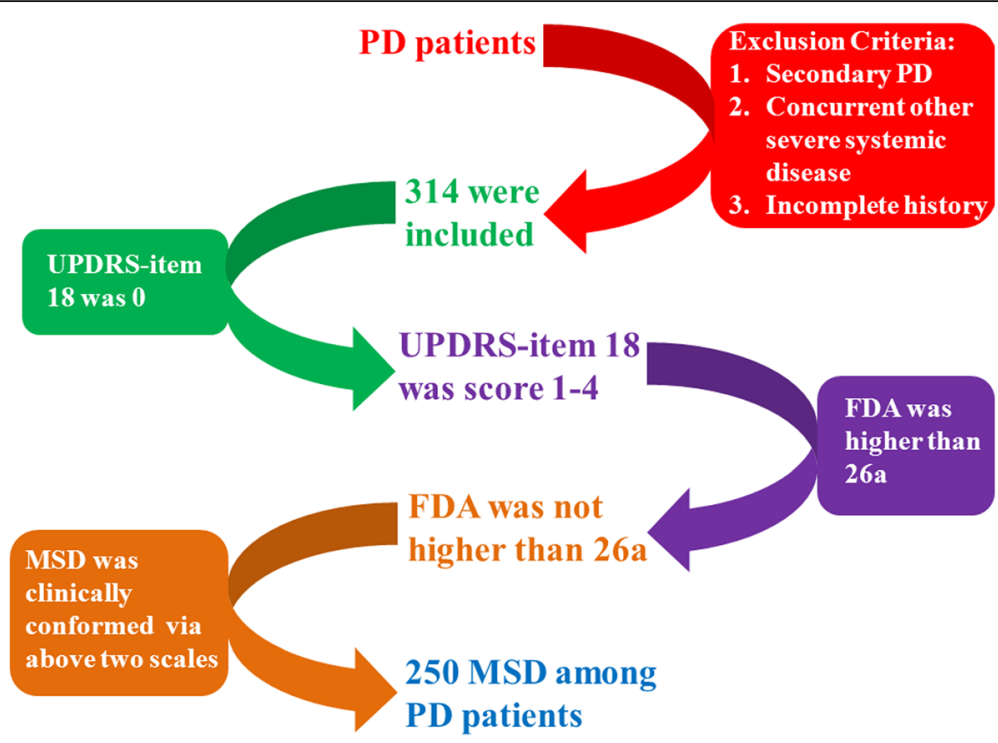

Fig. 1 The procedure of patient inclusion

the medical records of each patient. Hypertension was defined as the systolic pressure was higher than $140 \mathrm{mmHg}$ or (and) the diastolic pressure was higher than $90 \mathrm{mmHg}$, or a reported history of hypertension. Diabetes was defined as the fasting blood glucose was higher than 7.0 $\mathrm{mmol} / \mathrm{L}$ or (and) the 2-h postprandial blood pressure was higher than $11.1 \mathrm{mmol} / \mathrm{L}$, or a reported history of diabetes. Ischemic heart disease was defined as the change of ST-T segment in electrocardiogram plus a manifestation of angina pectoris, or a reported history of coronary heart disease. Lesions in the frontal or temporal lobe were defined as hypointense lesions on T1-weight and/or hyperintense lesions on T2-weight in the frontal or temporal lobe. According to the imaging principle of MRI, only such lesions that exceeded $1 \mathrm{~cm}$ in radius were counted. These neuroimaging data was evaluated by our two neurologists independently. Only when the two neurologists concurred that there was lesion in the frontal or temporal lobe could we draw a conclusion.

After obtaining access to each patient's case history, we evaluated the severity of PD using the modified Hoehn \& Yahr staging scale based on the corresponding clinical manifestations. Daily living ability was evaluated via the Modified Barthel Index of activities of daily living (ADL).

We comprehensively evaluated motor function, tremor, and postural instability gait difficulty (PIGD) using the UPDRS motor sum score, tremor score, and PIGD score, respectively [20].

The widely acknowledged NMSS was used to evaluate the degree of the NMM burden [21]. The NMSS contains a total of thirty items that are divided into nine domains that cover different aspects of daily living functions (i.e., cardiovascular disease, sleep/fatigue, mood/apathy, perceptual problems/hallucinations, attention/ memory, gastrointestinal, urinary, sexual function, and miscellaneous). Every item was evaluated quantitatively based on the severity (range: 0 to 3 ) and frequency (range: 0 to 4 ) over the previous month. The total score of each item was defined as the domain score. The total score of the NMSS, which is indicative of the overall NMM burden on each patient, was regarded as the sum of the thirty items.

\section{Statistical analysis}

All statistical analyses were carried out using SPSS software (version 18.0). Measurement data and continuous variables are presented as the mean and standard deviation or median and interquartile range, while enumeration data and categorical variables are presented as the frequency and proportion. For univariate analysis, we compared the measurement data from the MSD and non-MSD groups using t-tests or Mann-Whitney $U$ tests. Additionally, we used the Chi-squared or Fisher's exact tests to compare the enumeration data between the MSD and non-MSD groups.

We then carried out multivariate logistic regression analysis to determine the independent risk factors and rule out potential confounding variables. At first, all the factors with $P$-values lower than 0.05 in the t-tests, Mann-Whitney $U$ tests and Chi-squared tests were included. To reduce potential family wise error rate (FWER), namely type I error attributable to t-tests and Mann-Whitney $U$ tests, a Bonferroni correction of $P$ value was then used for statistical significance in the multivariate logistic regression analysis. After the final logistic regression analysis model, we only considered 
the risk factors with $P$-values lower than the corrected $P$-value to be the independent risk factors.

\section{Results}

\section{Demographic characteristics}

The demographic data of the two groups are shown in Table 1. There were 250 patients in the MSD group (168 males and 82 females) and 64 patients in the nonMSD group (40 males and 24 females). The average age was $64.3 \pm 9.5$ years in the MSD group and $62.3 \pm 8.7$ years in the non-MSD group. Student's $t$-test analysis

Table 1 The comparison of demographic characteristic between MSD and non-MSD groups

\begin{tabular}{llll}
\hline & MSD group & non-MSD group & $P$ value \\
\hline Age (years) & $64.3 \pm 9.5$ & $62.3 \pm 8.7$ & 0.088 \\
Age at PD onset (years) & $60.5 \pm 9.5$ & $58.4 \pm 8.6$ & 0.063 \\
Duration of PD (years) & $3.9 \pm 1.6$ & $4.0 \pm 1.6$ & 0.445
\end{tabular}

\begin{tabular}{llll}
\multicolumn{4}{c}{ PD family history (\%) } \\
Yes & $15(6.00 \%)$ & $7(10.94 \%)$ & 0.167 \\
No & $235(94.00 \%)$ & $57(89.06 \%)$ &
\end{tabular}

\begin{tabular}{llll}
\multicolumn{4}{c}{ Gender (\%) } \\
Male & $168(67.20 \%)$ & $40(62.50 \%)$ & 0.478 \\
Female & $82(32.80 \%)$ & $24(37.50 \%)$ &
\end{tabular}

\begin{tabular}{|c|c|c|c|}
\hline \multicolumn{4}{|c|}{ Marital status (\%) } \\
\hline Married & 238(95.20\%) & $58(90.63 \%)$ & 0.160 \\
\hline Unmarried & $12(4.80 \%)$ & $6(9.37 \%)$ & \\
\hline
\end{tabular}

$\begin{array}{llll}\text { Manual labor } & 170(68.00 \%) & 34(53.13 \%) & 0.026^{*} \\ \text { Mental labor } & 80(32.00 \%) & 30(46.87 \%) & \end{array}$

\begin{tabular}{cccc}
\multicolumn{5}{c}{ Educational level (\%) } & \\
Higher than bachelor & $40(16.00 \%)$ & $26(40.63 \%)$ & $<0.001^{*}$ \\
Lower than bachelor & $210(84.00 \%)$ & $38(59.37 \%)$ &
\end{tabular}

\begin{tabular}{llll}
\multicolumn{4}{c}{ Speaking background (\%) } \\
Monolingual speakers & $199(79.60 \%)$ & $58(90.62 \%)$ & $0.041^{*}$ \\
Bilingual speakers & $51(20.40 \%)$ & $6(9.38 \%)$ &
\end{tabular}

\begin{tabular}{|c|c|c|c|}
\hline \multicolumn{4}{|c|}{ Monthly income (yuan) } \\
\hline Higher than 5500 & $74(29.60 \%)$ & $28(43.75 \%)$ & $0.031^{*}$ \\
\hline Lower than 5500 & 176(70.40\%) & $36(56.25 \%)$ & \\
\hline \multicolumn{4}{|c|}{ Leisure activities (\%) } \\
\hline Usually & $123(49.20 \%)$ & $35(54.69 \%)$ & 0.433 \\
\hline Seldom & $127(50.80 \%)$ & $29(45.31 \%)$ & \\
\hline
\end{tabular}

showed that there was no significant difference in the average age, age at PD onset, and duration of PD between the two groups (all $P>0.05$ ). The proportion of patients with a family history of PD was similar in both groups $(P>0.05)$.

The Chi-squared test revealed that a higher percentage of patients engaged in manual labor in the MSD group $(68.00 \%)$ than in the non-MSD group $(53.13 \% ; P=$ $0.026)$. More patients in the MSD group $(84.00 \%)$ had a low level of education (lack of post-secondary education in university) than patients in the non-MSD group (59.37\%; $P<0.001)$. A higher percentage of patients were bilingual speakers in the MSD group (20.40\%) than in the non-MSD group $(9.38 \% ; P=0.041)$. More patients in the MSD group (70.40\%) had a low income (lower than 5500 yuan) compared to those in the non-MSD group (56.25\%; $P=0.031$ ). However, there were no significant differences between these two groups in terms of gender, marital status, or leisure activities $(P>0.05$ for all).

\section{Clinical characteristics}

Data on the clinical characteristics of the two groups are shown in Table 2. There were no significant differences between the two groups in terms of hypertension, hyperlipidemia, ischemic heart disease, or diabetes mellitus $(P>0.05$ for all).

The Chi-squared test revealed that there were higher incidence of frontal lobe lesions in the MSD group (78.00\%) than in the non-MSD group (59.38\%; $P=0.002)$. Similarly, there were higher incidence of temporal lobe lesions in the MSD group (51.60\%) than in the non-MSD group (35.94\%; $P=0.025)$. In addition, a higher percentage of patients had concurrent dysphagia in the MSD group $(46.40 \%)$ than in the non-MSD group $(31.25 \%$; $P=0.029)$. However, no significant differences were found in terms of parietal lobe lesion, occipital lobe lesions, concurrence with other CNS diseases and tremor dominant forms between these two groups $(P>0.05$ for all).

Anti-PD drugs, such as Levodopa, selegiline, and amantadine, were prescribed more often in the MSD group, while dopamine agonists were prescribed more often in the non-MSD group. However, there were no significant differences in the medication frequency or Levodopa equivalent dose between the two groups $(P>$ 0.05 for all).

\section{PD-associated evaluations}

The differences in the PD-associated evaluations between the two groups are shown in Table 3. The MannWhitney $U$ test revealed that the ADL score was lower in the MSD group than in the non-MSD group $(P=$ $0.020)$. However, there was no significant difference in the Hoehn \& Yahr stage score and duration of PD between the MSD group and non-MSD group $(P=0.095)$. 
Table 2 The comparison of clinical characteristic between MSD and non-MSD groups

\begin{tabular}{|c|c|c|c|}
\hline & MSD group & non-MSD group & $P$ value \\
\hline \multicolumn{4}{|c|}{ Hypertension (\%) } \\
\hline Yes & $172(68.80 \%)$ & $47(73.44 \%)$ & \multirow[t]{2}{*}{0.471} \\
\hline No & $78(31.20 \%)$ & $17(26.56 \%)$ & \\
\hline \multicolumn{4}{|c|}{ Hyperlipidemia (\%) } \\
\hline Yes & $94(37.60 \%)$ & $21(32.81 \%)$ & \multirow[t]{2}{*}{0.478} \\
\hline No & $156(62.40 \%)$ & $43(67.19 \%)$ & \\
\hline \multicolumn{4}{|c|}{ Ischemic heart disease (\%) } \\
\hline Yes & $33(13.20 \%)$ & $5(7.81 \%)$ & \multirow[t]{2}{*}{0.238} \\
\hline No & $217(86.80 \%)$ & 59(92.19\%) & \\
\hline \multicolumn{4}{|c|}{ Diabetes mellitus (\%) } \\
\hline Yes & $38(15.20 \%)$ & $12(18.75 \%)$ & \multirow[t]{2}{*}{0.489} \\
\hline No & $212(84.80 \%)$ & $52(81.25 \%)$ & \\
\hline \multicolumn{4}{|c|}{ Lesion in frontal lobe (\%) } \\
\hline Yes & 195(78.00\%) & $38(59.38 \%)$ & \multirow[t]{2}{*}{$0.002^{*}$} \\
\hline No & $55(22.00 \%)$ & $26(40.62 \%)$ & \\
\hline \multicolumn{4}{|c|}{ Lesion in temporal lobe (\%) } \\
\hline Yes & $129(51.60 \%)$ & $23(35.94 \%)$ & \multirow[t]{2}{*}{$0.025^{*}$} \\
\hline No & $121(48.40 \%)$ & $41(64.06 \%)$ & \\
\hline \multicolumn{4}{|c|}{ Lesion in parietal lobe (\%) } \\
\hline Yes & $2(0.80 \%)$ & $1(1.56 \%)$ & \multirow[t]{2}{*}{0.576} \\
\hline No & 248(99.20\%) & 63(98.44\%) & \\
\hline \multicolumn{4}{|c|}{ Lesion in occipital lobe (\%) } \\
\hline Yes & $1(0.40 \%)$ & $1(1.56 \%)$ & \multirow[t]{2}{*}{0.297} \\
\hline No & 249(99.60\%) & 63(98.44\%) & \\
\hline \multicolumn{4}{|c|}{ Concurrent dysphagia (\%) } \\
\hline Yes & $116(46.40 \%)$ & $20(31.25 \%)$ & \multirow[t]{2}{*}{$0.029 *$} \\
\hline No & $134(53.60 \%)$ & $44(68.75 \%)$ & \\
\hline \multicolumn{4}{|c|}{ Concurrent other CNS diseases (\%) } \\
\hline Yes & $54(21.60 \%)$ & $18(28.13 \%)$ & \multirow[t]{2}{*}{0.268} \\
\hline No & 196(78.40\%) & $46(71.87 \%)$ & \\
\hline \multicolumn{4}{|c|}{ Tremor dominant forms (\%) } \\
\hline Yes & $173(69.20 \%)$ & $41(64.06 \%)$ & \multirow[t]{2}{*}{0.431} \\
\hline No & $77(30.80 \%)$ & $23(35.94 \%)$ & \\
\hline \multicolumn{4}{|c|}{ Levodopa medication (\%) } \\
\hline Yes & $235(94.00 \%)$ & $58(90.63 \%)$ & \multirow[t]{2}{*}{0.335} \\
\hline No & $15(6.00 \%)$ & $6(9.37 \%)$ & \\
\hline \multicolumn{4}{|c|}{ Dopamine agonist medication (\%) } \\
\hline Yes & $237(94.80 \%)$ & $62(96.88 \%)$ & \multirow[t]{2}{*}{0.487} \\
\hline No & $13(5.20 \%)$ & $2(3.12 \%)$ & \\
\hline & & & \\
\hline Yes & $167(66.80 \%)$ & $39(60.94 \%)$ & 0.378 \\
\hline
\end{tabular}


Table 2 The comparison of clinical characteristic between MSD and non-MSD groups (Continued)

\begin{tabular}{llll}
\hline & MSD group & non-MSD group & $P$ value \\
\hline No & $83(33.20 \%)$ & $25(39.06 \%)$ \\
Amantadine medication (\%) & & \\
Yes & $228(91.20 \%)$ & $56(87.50 \%)$ \\
No & $22(8.80 \%)$ & $8(12.50 \%)$ \\
Levodopa equivalent dose $(\mathrm{mg})$ & 396.60 & 384.40 \\
\hline The "*" mark denotes a significant $P$ value $(P<0.05)$ after a statistical analysis & 0.369 \\
\end{tabular}

Regarding the UPDRS score, the MDS-UPDRS part III, UPDRS tremor score, and UPDRS PIGD scores were numerically, but not significantly, higher in the MSD group compared to the non-MSD group after the MannWhitney $U$ test $(P>0.05$ for all).

In the univariate analysis of the NMSS scores, the Mann-Whitney $U$ test revealed that both NMSS domain 4 (perceptual problem) and NMSS domain 5 (attention/ memory) scores were significantly higher in the MSD group than in the non-MSD group $(P=0.033$ and $P<$ 0.001 , respectively). However, there were no significant differences in the other NMSS domain scores between the two groups $(P>0.05$ for all $)$.

\section{Multivariate logistic regression}

At first, 10 significant factors resulted from the t-tests, Mann-Whitney $U$ tests and Chi-squared tests, including educational level, occupation, monthly income, speaking background, frontal lobe lesions, temporal lobe lesions, concurrent dysphagia, ADL score, NMSS domain 4 score (perceptual problems) and NMSS domain 5 score (attention/ memory) were included for multivariate logistic regression analysis. To reduce the potential FWER attributable to ttests and Mann-Whitney $U$ tests, a Bonferroni correction of $P$-value $(0.05 / 10=0.005)$ was reviewed to be a statistical significance. After the multivariate logistic regression analysis, 7 factors were excluded from the pool of independent risk factors. On the other hand, potential risk factors, including educational level, frontal lobe lesions, and NMSS domain 5 score (attention/memory), had a significant power to predict risk for MSD in PD (see Table 4).

The patients with a higher educational level [OR= $0.674,95 \%$ CI $(0.590,0.808)]$ had a lower likelihood of PD-associated MSD. However, patients with a higher occurrence of frontal lobe lesions $[\mathrm{OR}=5.145,95 \% \mathrm{CI}$ (2.018, 7.308)], or higher NMSS domain 5 scores (attention/memory) $[\mathrm{OR}=10.458,95 \% \mathrm{CI}(6.164,15.209)]$ had a higher likelihood of PD-associated MSD.

\section{Discussion}

This study comprehensively explored the independent risk factors, including demographic and clinical characteristics and relevant PD-associated MSD evaluations for the first time. Univariate analysis demonstrated that the

Table 3 The comparison of PD-associated evaluations between MSD and non-MSD groups

\begin{tabular}{llll}
\hline & MSD group & non-MSD group & $P$ value \\
\hline Hoehn\&Yahr stage score (median) & $2.0(1.0,3.0)$ & $2.0(1.0,2.5)$ & 0.095 \\
ADL score (median) & $75(70,80)$ & $80(70,85)$ & $0.020^{*}$ \\
MDS-UPDRS part III & $19.0(10.0,31.6)$ & $17.0(11.0,28.8)$ & 0.301 \\
UPDRS tremor score & $2.0(1.0,4.0)$ & $1.5(0,4.0)$ & 0.263 \\
UPDRS PIGD score & $2.0(0.5,4.0)$ & $1.5(0,3.0)$ & 0.320 \\
NMSS total score & $26.5(14.0,48.0)$ & $24.5(13.0,36.0)$ & 0.336 \\
NMSS domain 1 score (cardiovascular) & $0(0,1.0)$ & $0(0,1.0)$ & 0.249 \\
NMSS domain 2 score (sleep/fatigue) & $3.0(1.0,8.0)$ & $3.0(1.0,6.0)$ & 0.606 \\
NMSS domain 3 score (mood/apathy) & $4.0(1.0,12.0)$ & $4.0(1.0,11.0)$ & 0.743 \\
NMSS domain 4 score (perceptual problem) & $3.0(1.0,5.0)$ & $2.0(0,3.0)$ & $1.0(0,3.0)$ \\
NMSS domain 5 score (attention/memory) & $6.0(3.0,8.0)$ & $1.0(0,2.0)$ & $0.033^{*}$ \\
NMSS domain 6 score (gastrointestinal) & $1.0(0,3.0)$ & $2.0(0,6.0)$ & $0.001^{*}$ \\
NMSS domain 7 score (urinary) & $3.0(0.5,7.0)$ & $0(0,1.0)$ & 0.427 \\
NMSS domain 8 score (sexual function) & $0(0,0.1)$ & $1.0(0,4.0)$ & 0.126 \\
NMSS domain 9 score (miscellaneous) & $1.0(0,4.0)$ & & 0.802 \\
\hline
\end{tabular}

The "** mark denotes a significant $P$ value $(P<0.05)$ after a Mann-Whitney test 
Table 4 Potential independent risk factors for PD-associated MSD after the multivariate logisticregression analysis

\begin{tabular}{lll}
\hline Variables & $\mathrm{OR}(95 \% \mathrm{Cl})$ & $P$ value \\
\hline Educational level & $0.674(0.590,0.808)$ & 0.002 \\
Frontal lobe lesions & $5.145(2.018,7.308)$ & 0.004 \\
NMSS domain 5 score (attention/memory) & $10.458(6.164,15.209)$ & 0.001 \\
\hline
\end{tabular}

MSD phenotype was associated with a lower educational level and a greater amount of manual labor, and a monolingual speaking background. These findings were partially in accordance with those of similar studies that focused mainly on the demographic characteristics and relevant evaluations in other types of SD [22-25]. Moreover, multivariate logistic regression analysis revealed a lower educational level as an independent risk factor for PD-associated MSD. Several studies on the neural mechanisms underlying motor speech control $[15,16,26,27]$ have hypothesized that a higher educational level might play a negative role in the occurrence and aggravation of PD-associated MSD. Previous MRI examinations have shown that lesions in cognitive-relevant cortical areas, especially those closely associated with educational level within the prefrontal lobe, frequently occur in SD patients [28-30]. Given that educational level is usually positively associated with high-level cognitive function, this finding might partially explain why there were more PD patients with low educational levels in the MSD group in the present study [31-34].

Additionally, both univariate and multivariate logistic regression analyses revealed a higher occurrence of frontal lesions in the MSD group, indicating that frontal lesions are another important independent risk factor for PD-associated MSD. This is could also explain our speculation that PD patients in the MSD group were more prone to cognitive impairment since frontal lesions have been reported as a risk factor for the development of cognitive dysfunction [35-38]. Additionally, the higher occurrence of frontal lesions in the MSD group might also be attributed to a lack of sufficient plastic repair in some cortical areas following PD, especially in the frontal lobe, a well-known key area for central modulation during speech motor processing. On the other hand, secondary damage remote from the basal ganglia, an indispensible fiber pathway and subcortical interconnecting structure, could occur in the frontal lobe [39-41]. Therefore, future prospective clinical and neuroimaging studies are necessary to confirm these relationships and provide new insights into the neuroplastic repair in cortical areas.

Previous studies have systematically explored the relationship between MSD and cardinal motor symptoms. Moustafa et al. found that motor symptoms such as gait disturbance share similar clinical profiles and neural bases with MSD [42]. Majdinasab et al. found that tremor was the only aspect of motor symptoms that influenced MSD among PD patients [43]. However, in the present study, the UPDRS motor sum, tremor, and PIGD score were all ruled out following the univariate analysis. The difference between our study and that of Majdinasab et al. might be in the different disease stages. Patients in the previous study were predominantly in the early stage of PD, when motor symptoms are common, while our patients were in more progressive stages of PD. Additionally, both MSD and other motor symptoms are partly under the modulation of the extrapyramidal system, which causes similar neuropathological changes.

In the present study, univariate analysis showed that the NMM burden was slightly greater in the MSD group than in the non-MSD group, which was consistent with our speculation that MSD and NMM share similar neuropathological mechanisms. We found that the MSD group had a higher NMMSS domain 5 score (attention/ memory) than the non-MSD group. Cognitive function impairment, including attention, memory, and executive functions, are common in PD patients; therefore, the cause and effect relationship between cognitive impairment and MSD requires in-depth examination. In terms of neural substrates, the frontal lobe is predominantly considered as the key modulation area responsible for the neural coding of high-level cognitive function and as a positive influencer of cognitive integration, which explains the attention and memory impairment in the MSD group [44-46]. In addition to the anatomical findings, we also speculate that MSD and cognitive impairment might share a similar pathophysiological process. Consistent with several previous findings, we found that the invalidation of anti-PD medication was more remarkable in PD patients who developed MSD and other NMMs than in tremor-dominant patients. These findings indicate that the root cause of MSD and other NMMs might involve non-dopaminergic neurons and non-dopaminergic transmitter disorders, such as longterm neurodegeneration induced by a gradual loss of cholinergic neurons [10, 12, 47]. Generally, we reasoned that the MSD group was likely to experience more cognitive dysfunction since speech motor modulation and cognitive functions are likely to share common neural anatomy and neurodegenerative processes. In the present study, attention/memory impairment was retained after removal of the potential risk factor (perceptual problems) from the multivariate logistic regression analysis, suggesting that 
there may be a strong correlation between high-level cognitive function (especially attention and memory) and MSD in PD patients. This finding demonstrated that a detailed scale for neuroimaging evaluation of attention and memory, as well as valid cognitive treatment for the improvement of attention and memory, might improve the prognosis of MSD in PD patients. Moreover, determining the cause and effect relationship between MSD and attention/memory impairment in PD could help determine the detailed neurophysiologic and neuropathological mechanisms underlying the abnormal speech motor modulation. In addition, although we evaluated attention and memory as two separate high-level cognitive functions, it was difficult to separate the two different cognitive subtypes in the MSD and nonMSD groups. This could be attributed to the fact that a majority of the patients manifested with mixed dysfunction of the two cognitive subtypes, or that speech modulation involves the two cognitive subtypes. Nonetheless, we have to acknowledge that we just assessed patients' attention or memory capacity via a single subjective question in NMSS, respectively. Therefore, lack of some direct objective cognition tests might bring about some limitation to present study. In the future study, we plan to carry out some direct objective cognition measurements to further explore the potential relationship between cognition and MSD in PD patients.

Several different studies have reported that speech is a multisensory process in which auditory perception plays a key role [48-51]. Additionally, some studies have reported that MSD patients are more likely to suffer more from perceptual disorder; however, the elaborate relationship and mechanism involved in PD-associated perceptual problems are unknown [52-54]. In the present study, univariate analysis showed a greater burden of perceptual problems in the MSD group than in the nonMSD group, but multivariate logistic regression analysis excluded perceptual problems as an independent risk factor for PD-associated MSD. However, as in the previous study, we did not explicitly define perceptual problems based on specific sensory function disorders. Therefore, studies with larger sample sizes and more comprehensive methods for evaluation of specific sensory disorders are necessary to confirm the relationship between perceptual problems and PD-associated MSD and to determine if more severe perceptual problems are related to a higher likelihood of MSD in PD patients.

\section{Limitations}

Tough we have tried our best to improve our present study, there are still some limitation as follows. Firstly, as mentioned in Discussion section, we just evaluated attention/memory capacity by a single item subjective question, respectively. This might make our study less objective, in that cognition tests were missing. Secondly, there was a little shortage in our neuroimaging method. We have successfully prevented missing MRI data, however, we just measured the occurrence of lesion within any a lobe rather than the number or the volume of lesions. This might make our present study ambiguous that we have not appropriately utilized our MRI data. It is helpful for our addition of limitation to readers that they will be more objective to interpret our present study.

\section{Conclusions}

We demonstrated more severe attention/memory function impairment in the MSD group compared to the nonMSD group. Additionally, for the first time, cognitive impairment was shown to be strongly correlated with MSD in PD patients. The early application of cognitive treatment may help improve rehabilitation management and quality of life in patients with PD-associated MSD. Moreover, future studies could further assess cognitive impairment to determine the detailed mechanisms underlying abnormal speech motor modulation in PD patients.

\section{Abbreviations \\ ADL: Activities of daily living; FDA: Frenchay dysarthria assessment; MRI: Magnetic resonance imaging; MSD: Motor speech disorder; NMM: Non- motor manifestations; PD: Parkinson's disease; PIGD: Postural instability gait difficulty; SD: Speech disorders; UPDRS: Unified Parkinson's Disease Rating Scale}

\section{Acknowledgements}

Not applicable.

\section{Authors' contributions}

JX and YLiu: Designed the experiment; YG, JH, SL, SM, YLi, FZ and YZ: Performed the investigation and analyzed the data; YLiu, YG, JH, YZ and JX: Interpreted the results and wrote the manuscript. All authors read and approved the final manuscript.

\section{Funding}

The present investigation was supported by the National Nature and Science Foundation of China (Nos.81901394 and 81960417), Guangxi Nature and Science Foundation (Nos. 2016GXNSFBA380020, 2018GXNSFFAA050033 and 2018GXNSFAA138010). The roles of funding body were played in the design of the study, collection, analysis, and interpretation of data.

\section{Availability of data and materials}

The datasets used and/or analysed during the current study are available from the corresponding author on reasonable request.

\section{Ethics approval and consent to participate}

The present investigation was officially approved by the ethics committee of the First Affiliated Hospital of Guangxi Medical University. All patients have provided the written informed consent for participation in this investigation.

\section{Consent for publication}

Written informed consent was obtained from each patient. The consent was for publication of the demographic information, medical history, current manifestation, radiological information and evaluated results.

\section{Competing interests}

The authors declare that they have no competing interests.

\section{Author details}

${ }^{1}$ Department of Rehabilitation Medicine, the First Affiliated Hospital of Guangxi Medical University, Nanning 530021, Guangxi, China. ${ }^{2}$ Department 
of Neurology, the First Affiliated Hospital of Guangxi Medical University, Nanning 530021, Guangxi, China.

\section{Received: 22 October 2019 Accepted: 19 November 2019 Published online: 01 December 2019}

\section{References}

1. Hirsch L, Jette N, Frolkis A, Steeves T, Pringsheim T. The incidence of Parkinson's disease: a systematic review and meta-analysis. Neuroepidemiology. 2016;46(4):292-300. https://doi.org/10.1159/000445751.

2. Huang X, Ng SY, Chia NS, Setiawan F, Tay KY, Au WL, Tan EK, Tan LC. Nonmotor symptoms in early Parkinson's disease with different motor subtypes and their associations with quality of life. Eur J Neurol. 2019;26(3):400-6. https://doi.org/10.1111/ene.13803.

3. Rosqvist $K$, Odin P, Hagell P, Iwarsson S, Nilsson MH, Storch A Dopaminergic effect on non-motor symptoms in late stage Parkinson's disease. J Park Dis. 2018;8(3):409-20. https://doi.org/10.3233/JPD-181380.

4. Kim A, Kim HJ, Shin CW, Kim A, Kim Y, Jang M, Jung YJ, Lee WW, Park H, Jeon B. Emergence of non-motor fluctuations with reference to motor fluctuations in Parkinson's disease. Parkinsonism Relat Disord. 2018:54:79-83. https://doi.org/10.1016/j.parkreldis.2018.04.020

5. Burn DJ, Landau S, Hindle JV, Samuel M, Wilson KC, Hurt CS, Brown RG, Group P-PS. Parkinson's disease motor subtypes and mood. Mov Disord. 2012;27(3):379-86. https://doi.org/10.1002/mds.24041.

6. New AB, Robin DA, Parkinson AL, Eickhoff CR, Reetz K, Hoffstaedter F, Mathys C, Sudmeyer M, Grefkes C, Larson CR, Ramig LO, Fox PT, Eickhoff SB. The intrinsic resting state voice network in Parkinson's disease. Hum Brain Mapp. 2015;36(5):1951-62. https://doi.org/10.1002/hbm.22748.

7. Theodoros DG, Hill AJ, Russell TG. Clinical and quality of life outcomes of speech treatment for Parkinson's disease delivered to the home via Telerehabilitation: a noninferiority randomized controlled trial. Am J Speech Lang Pathol. 2016;25(2):214-32. https://doi.org/10.1044/2015_AJSLP-15-0005.

8. Bornkessel-Schlesewsky I, Schlesewsky M, Small SL, Rauschecker JP. Neurobiological roots of language in primate audition: common computational properties. Trends Cogn Sci. 2015;19(3):142-50. https://doi. org/10.1016/j.tics.2014.12.008

9. Small SL, Buccino G, Solodkin A. Brain repair after stroke--a novel neurological model. Nat Rev Neurol. 2013;9(12):698-707. https://doi.org/10. 1038/nrneurol.2013.222.

10. Fabbri M, Guimaraes I, Cardoso R, Coelho M, Guedes LC, Rosa MM, Godinho C, Abreu D, Goncalves N, Antonini A, Ferreira JJ. Speech and voice response to a levodopa challenge in late-stage Parkinson's disease. Front Neurol. 2017:8:432. https://doi.org/10.3389/fneur.2017.00432.

11. Elfmarkova N, Gajdos M, Mrackova M, Mekyska J, Mikl M, Rektorova I. Impact of Parkinson's disease and levodopa on resting state functional connectivity related to speech prosody control. Parkinsonism Relat Disord. 2016;22(Suppl 1):S52-5. https://doi.org/10.1016/j.parkreldis.2015.09.006.

12. Maillet A, Krainik A, Debu B, Tropres I, Lagrange C, Thobois S, Pollak P, Pinto S. Levodopa effects on hand and speech movements in patients with Parkinson's disease: a FMRI study. PLoS One. 2012;7(10):e46541. https://doi. org/10.1371/journal.pone.0046541.

13. Franken MK, Eisner F, Acheson DJ, McQueen JM, Hagoort P, Schoffelen JM Self-monitoring in the cerebral cortex: neural responses to small pitch shifts in auditory feedback during speech production. Neuroimage. 2018;179:32636. https://doi.org/10.1016/j.neuroimage.2018.06.061.

14. Behroozmand R, Phillip L, Johari K, Bonilha L, Rorden C, Hickok G, Fridriksson J. Sensorimotor impairment of speech auditory feedback processing in aphasia. Neuroimage. 2018;165:102-11. https://doi.org/10.1016/j. neuroimage.2017.10.014

15. Hu H, Liu Y, Guo Z, Li W, Liu P, Chen S, Liu H. Attention modulates cortical processing of pitch feedback errors in voice control. Sci Rep. 2015;5:7812. https://doi.org/10.1038/srep07812

16. Guo Z, Wu X, Li W, Jones JA, Yan N, Sheft S, Liu P, Liu H. Top-down modulation of auditory-motor integration during speech production: the role of working memory. J Neurosci. 2017;37(43):10323-33. https://doi.org/ 10.1523/JNEUROSCI.1329-17.2017.

17. Cabeza R, Daselaar SM, Dolcos F, Prince SE, Budde M, Nyberg L. Taskindependent and task-specific age effects on brain activity during working memory, visual attention and episodic retrieval. Cereb Cortex. 2004;14(4): 364-75. https://doi.org/10.1093/cercor/bhg133.
18. Lunner T, Sundewallthoren E. Interactions between cognition, compression, and listening conditions: effects on speech-in-noise performance in a twochannel hearing aid. J Am Acad Audiol. 2007;18:604-17.

19. Gibb WR, Lees AJ. The relevance of the Lewy body to the pathogenesis of idiopathic Parkinson's disease. J Neurol Neurosurg Psychiatry. 1988;51(6): 745-52. https://doi.org/10.1136/jnnp.51.6.745.

20. Goetz CG, Poewe W, Rascol O, Sampaio C, Stebbins GT, Counsell C, Giladi N, Holloway RG, Moore CG, Wenning GK, Yahr MD, Seidl L, Movement Disorder Society Task Force on Rating Scales for Parkinson's D. Movement Disorder Society task force report on the Hoehn and Yahr staging scale: status and recommendations. Mov Disord. 2004; 19(9):1020-8. https://doi.org/10.1002/mds.20213.

21. Chaudhuri KR, Martinez-Martin P, Brown RG, Sethi K, Stocchi F, Odin P Ondo W, Abe K, Macphee G, Macmahon D, Barone P, Rabey M, Forbes A, Breen K, Tluk S, Naidu Y, Olanow W, Williams AJ, Thomas S, Rye D, Tsuboi Y, Hand A, Schapira AH. The metric properties of a novel non-motor symptoms scale for Parkinson's disease: results from an international pilot study. Mov Disord. 2007;22(13):1901-11. https://doi.org/10.1002/mds.21596.

22. Yun C, Wang Z, He P, Guo C, Chen G, Zheng X. Prevalence and parental risk factors for speech disability associated with cleft palate in Chinese childrena National Survey. Int J Environ Res Public Health. 2016;13(11). https://doi. org/10.3390/ijerph13111168.

23. Seifpanahi S, Izadi F, Jamshidi AA, Torabinezhad F, Sarrafzadeh J, SobhaniRad D, Ganjuie M. Prevalence of voice disorders and associated risk factors in teachers and nonteachers in Iran. J Voice. 2016;30(4):506.e519-23. https:// doi.org/10.1016/j.jvoice.2015.05.019.

24. Korpilahti $P$, Kaljonen A, Jansson-Verkasalo E. Identification of biological and environmental risk factors for language delay: the Let's talk STEPS study. Infant Behav Dev. 2016;42:27-35. https://doi.org/10.1016/j.infbeh. 2015.08.008.

25. Ward D. Risk factors and stuttering: evaluating the evidence for clinicians. J Fluen Disord. 2013;38(2):134-40. https://doi.org/10.1016/j.jludis.2013.02.007.

26. Liu Y, Hu H, Jones JA, Guo Z, Li W, Chen X, Liu P, Liu H. Selective and divided attention modulates auditory-vocal integration in the processing of pitch feedback errors. Eur J Neurosci. 2015;42(3):1895-904. https://doi.org/ 10.1111/ejn.12949.

27. Liu Y, Fan H, Li J, Jones JA, Liu P, Zhang B, Liu H. Auditory-motor control of vocal production during divided attention: behavioral and ERP correlates. Front Neurosci. 2018;12:113. https://doi.org/10.3389/fnins.2018.00113.

28. Conant LL, Liebenthal E, Desai A, Binder JR. The relationship between maternal education and the neural substrates of phoneme perception in children: interactions between socioeconomic status and proficiency level. Brain Lang. 2017;171:14-22. https://doi.org/10.1016/j.bandl.2017.03.010.

29. Lieu JE. Speech-language and educational consequences of unilateral hearing loss in children. Arch Otolaryngol Head Neck Surg. 2004;130(5):52430. https://doi.org/10.1001/archotol.130.5.524.

30. Le Dorze G, Bedard C. Effects of age and education on the lexico-semantic content of connected speech in adults. J Commun Disord. 1998;31(1):53-70 quiz 71.

31. Patra K, Greene MM, Patel AL, Meier P. Maternal education level predicts cognitive, language, and motor outcome in preterm infants in the second year of life. Am J Perinatol. 2016;33(8):738-44. https://doi.org/10.1055/s0036-1572532.

32. Xie H, Zhang C, Wang Y, Huang S, Cui W, Yang W, Koski L, Xu X, Li Y, Zheng M, He M, Fu J, Shi X, Wang K, Tang G, Wang B, Huo Y. Distinct patterns of cognitive aging modified by education level and gender among adults with limited or no formal education: a normative study of the mini-mental state examination. J Alzheimers Dis. 2016;49(4):961-9. https://doi.org/10. 3233/JAD-143066

33. Castanho TC, Portugal-Nunes C, Moreira PS, Amorim L, Palha JA, Sousa N, Correia Santos N. Applicability of the telephone interview for cognitive status (modified) in a community sample with low education level: association with an extensive neuropsychological battery. Int J Geriatr Psychiatry. 2016:31(2):128-36. https://doi.org/10.1002/gps.4301.

34. Yancar Demir E, Ozcan T. Evaluating the relationship between education level and cognitive impairment with the Montreal cognitive assessment test. Psychogeriatrics. 2015;15(3):186-90. https://doi.org/10.1111/psyg.12093.

35. Leonard MK, Desai M, Hungate D, Cai R, Singhal NS, Knowlton RC, Chang EF. Direct cortical stimulation of inferior frontal cortex disrupts both speech and music production in highly trained musicians. Cogn Neuropsychol. 2018:1-9. https://doi.org/10.1080/02643294.2018.1472559. 
36. Khoshkhoo S, Leonard MK, Mesgarani N, Chang EF. Neural correlates of sine-wave speech intelligibility in human frontal and temporal cortex. Brain Lang. 2018;187:83-91. https://doi.org/10.1016/j.bandl.2018.01.007.

37. Zhao W, Riggs K, Schindler I, Holle H. Transcranial magnetic stimulation over left inferior frontal and posterior temporal cortex disrupts gesture-speech integration. J Neurosci. 2018;38(8):1891-900. https://doi.org/10.1523/ JNEUROSCI.1748-17.2017.

38. Cope TE, Sohoglu E, Sedley W, Patterson K, Jones PS, Wiggins J, Dawson C, Grube M, Carlyon RP, Griffiths TD, Davis MH, Rowe JB. Evidence for causal top-down frontal contributions to predictive processes in speech perception. Nat Commun. 2017;8(1):2154. https://doi.org/10.1038/s41467017-01958-7.

39. Tang X, Chen N, Zhang S, Jones JA, Zhang B, Li J, Liu P, Liu H. Predicting auditory feedback control of speech production from subregional shape of subcortical structures. Hum Brain Mapp. 2018;39(1):459-71. https://doi.org/ 10.1002/hbm.23855.

40. Huang X, Chen X, Yan N, Jones JA, Wang EQ, Chen L, Guo Z, Li W, Liu P, Liu $H$. The impact of parkinson's disease on the cortical mechanisms that support auditory-motor integration for voice control. Hum Brain Mapp. 2016;37(12):4248-61. https://doi.org/10.1002/hbm.23306.

41. Chen X, Zhu X, Wang EQ, Chen L, Li W, Chen Z, Liu H. Sensorimotor control of vocal pitch production in Parkinson's disease. Brain Res. 2013;1527:99107. https://doi.org/10.1016/j.brainres.2013.06.030.

42. Moustafa AA, Chakravarthy S, Phillips JR, Gupta A, Keri S, Polner B, Frank MJ, Jahanshahi M. Motor symptoms in Parkinson's disease: a unified framework. Neurosci Biobehav Rev. 2016;68:727-40. https://doi.org/10.1016/..neubiorev. 2016.07.010.

43. Majdinasab F, Karkheiran S, Soltani M, Moradi N, Shahidi G. Relationship between voice and motor disabilities of Parkinson's disease. J Voice. 2016; 30(6):768.e717-22. https://doi.org/10.1016/j.jvoice.2015.10.022.

44. Mesgarani N, Chang EF. Selective cortical representation of attended speaker in multi-talker speech perception. Nature. 2012;485(7397):233-6. https://doi.org/10.1038/nature11020

45. Wong PC, Jin JX, Gunasekera GM, Abel R, Lee ER, Dhar S. Aging and cortical mechanisms of speech perception in noise. Neuropsychologia. 2009;47(3): 693-703. https://doi.org/10.1016/j.neuropsychologia.2008.11.032.

46. Rinne T, Pekkola J, Degerman A, Autti T, Jaaskelainen IP, Sams M, Alho K. Modulation of auditory cortex activation by sound presentation rate and attention. Hum Brain Mapp. 2005;26(2):94-9. https://doi.org/10.1002/hbm.20123.

47. Simuni T, Sethi K. Nonmotor manifestations of Parkinson's disease. Ann Neurol. 2008;64(Suppl 2):S65-80. https://doi.org/10.1002/ana.21472.

48. Liu X, Tian X. The functional relations among motor-based prediction, sensory goals and feedback in learning non-native speech sounds: evidence from adult mandarin Chinese speakers with an auditory feedback masking paradigm. Sci Rep. 2018;8(1):11910. https://doi.org/10. 1038/s41598-018-30399-5.

49. Ito T, Coppola JH, Ostry DJ. Speech motor learning changes the neural response to both auditory and somatosensory signals. Sci Rep. 2016;6: 25926. https://doi.org/10.1038/srep25926.

50. Max L, Maffett DG. Feedback delays eliminate auditory-motor learning in speech production. Neurosci Lett. 2015;591:25-9. https://doi.org/10.1016/j. neulet.2015.02.012

51. Scheerer NE, Jones JA. The predictability of frequency-altered auditory feedback changes the weighting of feedback and feedforward input for speech motor control. Eur J Neurosci. 2014;40(12):3793-806. https://doi.org/ 10.1111/ejn.12734

52. Iliadou W, Weihing J, Chermak GD, Bamiou DE. Otoacoustic emission suppression in children diagnosed with central auditory processing disorder and speech in noise perception deficits. Int J Pediatr Otorhinolaryngol. 2018; 111:39-46. https://doi.org/10.1016/j.jporl.2018.05.027.

53. Delphi M, Lotfi MY, Moossavi A, Bakhshi E, Banimostafa M. Reliability of Interaural time difference-based localization training in elderly individuals with speech-in-noise perception disorder. Iran J Med Sci. 2017;42(5):437-42.

54. Stevenson RA, Baum SH, Segers M, Ferber S, Barense MD, Wallace MT. Multisensory speech perception in autism spectrum disorder: from phoneme to whole-word perception. Autism Res. 2017;10(7):1280-90. https://doi.org/10.1002/aur.1776.

\section{Publisher's Note}

Springer Nature remains neutral with regard to jurisdictional claims in published maps and institutional affiliations.

Ready to submit your research? Choose BMC and benefit from:

- fast, convenient online submission

- thorough peer review by experienced researchers in your field

- rapid publication on acceptance

- support for research data, including large and complex data types

- gold Open Access which fosters wider collaboration and increased citations

- maximum visibility for your research: over $100 \mathrm{M}$ website views per year

At $\mathrm{BMC}$, research is always in progress.

Learn more biomedcentral.com/submissions 